\title{
SIMULAÇÃO TRIDIMENSIONAL DA DISPERSÃO DE POLUENTES EM CONDIÇÕES DE VENTO FRACO E ESTÁVEIS
}

\author{
Viliam Cardoso da Silveira ${ }^{*}$, Daniela Buske, Régis Sperotto de Quadros, Jonas Carvalho
}

Programa de Pós-graduação em Meteorologia - UFPel

*viliamcardoso@gmail.com

\section{RESUMO}

Neste trabalho apresentamos um modelo matemático para simular a dispersão de poluentes em condições estáveis e de vento fraco, que leva em conta a difusão longitudinal. A solução da equação de advecção-difusão tridimensional para estas condições é obtida utilizando o método 3D-GILTT (Three-dimensional Generalized Integral Laplace Transform Technique). Simulações numéricas e comparações com dados experimentais são apresentadas.

\begin{abstract}
The present study proposes a mathematical model for dispersion of contaminants in stable conditions and low winds, that takes into account the along-wind diffusion. The solution of the three-dimensional advection-diffusion equation for these conditions is obtained applying the 3D-GILTT method. Numerical results and comparison with experimental data are presented.
\end{abstract}

\section{A SOLUÇÃO ANALÍTICA}

Para simular a dispersão de poluentes na atmosfera em condições de vento fraco, consideramos neste trabalho a equação de advecção-difusão escrita da seguinte forma:

$$
\bar{u} \frac{\partial \bar{c}}{\partial x}=\frac{\partial}{\partial x}\left(K_{x} \frac{\partial \bar{c}}{\partial x}\right)+K_{y} \frac{\partial^{2} \bar{c}}{\partial y^{2}}+\frac{\partial}{\partial z}\left(K_{z} \frac{\partial \bar{c}}{\partial z}\right)
$$

Esta equação está sujeita as condições de contorno de fluxo nulo nas fronteiras $\left(K_{x} \frac{\partial \bar{c}\left(L_{x}, y, z\right)}{\partial x}=K_{y} \frac{\partial \bar{c}(x, 0, z)}{\partial y}=K_{y} \frac{\partial \bar{c}\left(x, L_{y}, z\right)}{\partial y}=K_{z} \frac{\partial \bar{c}(x, y, 0)}{\partial z}=K_{z} \frac{\partial \bar{c}(x, y, h)}{\partial z}=0\right)$ e a condição de fonte $\bar{u} \bar{c}(0, y, z)=Q \delta\left(y-y_{0}\right) \delta\left(z-H_{s}\right)$, onde $h$ é a altura da CLA, $Q$ é a taxa de emissão de poluentes, $L_{x}$ e $L_{y}$ são as distâncias para longe da fonte, $\bar{u}$ é o vento médio na direção $x$, $H_{s}$ é a altura de fonte, $K_{x}, K_{y}$ e $K_{z}$ são os coeficientes de difusão turbulenta nas direções $x, y$ e $z$, respectivamente e $\delta$ é a função delta de Dirac.

Para resolver o problema da Eq. (1), aplica-se a técnica da transformada integral na variável $y$, expandindo a concentração de poluentes da seguinte forma: 


$$
\bar{c}(x, y, z)=\sum_{n=0}^{N} \overline{c_{n}}(x, z) \zeta_{n}(y)
$$

Substituindo a Eq. (2) na Eq. (1) e tomando momentos, obtemos o seguinte conjunto de equações bidimensionais:

$$
\begin{array}{r}
\bar{u} \frac{\partial \overline{c_{n}}(x, z)}{\partial x}=\frac{\partial}{\partial x}\left(K_{x} \frac{\partial \overline{c_{n}}(x, z)}{\partial x}\right)+\frac{\partial}{\partial z}\left(K_{z} \frac{\partial \overline{c_{n}}(x, z)}{\partial z}\right) \\
-\lambda_{n}^{2} K_{y} \overline{c_{n}}(x, z)
\end{array}
$$

A Eq. (3) é resolvida pela técnica GILTT cuja equação transformada é uma equação diferencial ordinária matricial de segunda ordem que é resolvida analiticamente pelo método de redução de ordem, transformada de Laplace e diagonalização. Maiores detalhes são encontrados no trabalho (Buske et al., 2007).

\section{PARAMETRIZAÇÃO DA TURBULÊNCIA}

O coeficiente de difusão longitudinal não deve ser desprezado em condições de vento fraco e estáveis. Nestas condições os coeficientes de difusão devem ser considerados não somente como funções da turbulência, mas também da distância da fonte (Degrazia et al., 1996). Neste trabalho a expressão utilizada é escrita como:

$$
K_{\alpha}=\frac{2 \sqrt{\pi} 0.64 u_{*} h a_{i}^{2}(1-z / h)^{\alpha_{1}}(z / h) X^{*}\left[2 \sqrt{\pi} 0.64 a_{i}^{2}(z / h)+8 a_{i}\left(f_{m}\right)_{i}(1-z / h)^{\alpha_{1} / 2} X^{*}\right]}{\left[2 \sqrt{\pi} 0.64(z / h)+16 a_{i}\left(f_{m}\right)_{i}(1-z / h)^{\alpha_{1} / 2} X^{*}\right]^{2}}
$$

Detalhes sobre os parâmetros e variáveis podem ser encontrados em Degrazia et al. (1996). O perfil do vento utilizado é descrito por uma lei de potência (Panofsky, Dutton, 1984).

\section{RESULTADOS E DISCUSSÕES}

Nas simulações numéricas do modelo foram utilizados os dados observados sob condições de vento fraco no experimento estável de INEL (Sagendorf; Dickson, 1974). Na Figura 1a, podemos ver o gráfico de espalhamento dos dados observados no experimento versus os dados preditos pelo modelo apresentado. Na Figura $1 \mathrm{~b}$, podemos ver o gráfico de espalhamento considerando somente os casos em que o vento é menor que $1 \mathrm{~m} / \mathrm{s}$.
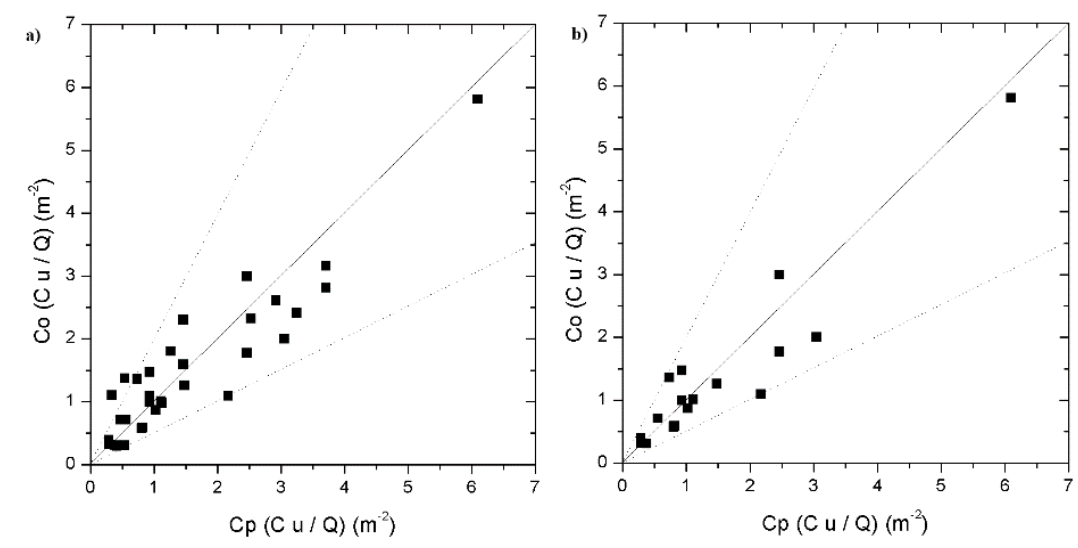

Figura 1: (a) Diagrama de espalhamento das concentrações observadas no experimento INEL e preditas pelo modelo 3D-GILTT. (b) idem (a) mas para $u<1 \mathrm{~m} / \mathrm{s}$. 
Os resultados estatísticos (Hanna, 1989) são apresentados na Tab. 1, onde é possível notar que o modelo simula de forma satisfatória as concentrações observadas, com os valores de NMSE, FB e FS relativamente próximo de zero e COR e FA2 relativamente próximo de 1. Melhores resultados são obtidos para o caso $u<1 \mathrm{~m} / \mathrm{s}$.

Tabela 1: Comparação estatística entre os resultados do modelo 3D-GILTT e os dados do experimento INEL.

\begin{tabular}{cccccc}
\hline Modelo & NMSE & COR & FA2 & FB & FS \\
\hline 3D-GILTT & 0.11 & 0.93 & 0.94 & -0.04 & -0.15 \\
\hline $\begin{array}{c}\text { 3D-GILTT } \\
(\mathrm{u}<1 \mathrm{~m} / \mathrm{s})\end{array}$ & 0.12 & 0.94 & 1.00 & -0.09 & -0.08 \\
\hline
\end{tabular}

\section{CONCLUSÕES}

O presente trabalho mostra que a inclusão da difusão longitudinal e coeficientes de difusão dependentes da distância da fonte, importante em condições de vento fraco, fornecem uma boa descrição do processo de transporte turbulento dos poluentes atmosféricos.

\section{AGRADECIMENTOS}

Os autores agradecem a CAPES, ao CNPq e a Fapergs pelo auxílio financeiro.

\section{REFERÊNCIAS BIBLIOGRÁFICAS}

Buske, D., Vilhena, M.T., Moreira, D.M., Tirabassi, T., 2007. Simulation of pollutant dispersion for low wind conditions in stable and convective planetary boundary layer. Atmos. Environ. 41, 5496-5501.

Degrazia, G.A., Vilhena, M.T., Moraes, O.L.L., 1996. An algebraic expression for the eddy diffusivities in the stable boundary layer: a description of near-source diffusion. IINuovoCimento 19C, 399-403.

Hanna, S.R., 1989. Confidence limit for air quality models as estimated by bootstrap and jacknife resampling methods. Atmos. Environ. 23, 1385-1395.

Panofsky, H.A., Dutton, J.A., 1984. Atmospheric Turbulence. New York: John Wiley \&Sons.

Sagendorf, J.F., Dickson, C.R., 1974.Diffusion under low wind-speed, inversion conditions. Technical Memorandum ERL ARL-52, U.S. National Oceanics and Atmospheric Administration. 